\title{
Impact of Instruction Booklet About Woman Rights in Reproductive Health on Developing Nursing Students Knowledge and Misconceptions
} \author{
Ahmed $^{4}$ \\ ${ }^{1}$ Department of Maternity, Obstetrics and Gynecology Nursing, Ismailia Technical Institute of Health, Egypt \\ ${ }^{2}$ Department of Community\& Family Health Nursing, Faculty of Nursing- Port said University, Egypt \\ ${ }^{3}$ Department of Community Health Nursing, Faculty of Nursing, Beni- Suef University, Egypt \\ ${ }^{4}$ Department of Maternal\& newborn Health Nursing, Faculty of Nursing, Beni- Suef University, Egypt
}

Nadra Mohamed Ibraheim ${ }^{1 *}$, Ebtesam EL Sayed Shama ${ }^{1}$, Naglaa Ghida ${ }^{2}$, Eman El-Sherbeny ${ }^{3}$ and Amal Roshdi

Submission: December 14, 2020; Published: December 22, 2020

*Corresponding author: Nadra Mohamed Ibraheim, Department of Maternity, Obstetrics and Gynecology Nursing, Ismailia Technical Institute of Health, Egypt

\begin{abstract}
Background: Reproductive health is an important component of general health and central feature of human development. It is a reflection of health during childhood, and crucial during adolescence and adulthood, sets the stage for health beyond the reproductive years for both women and men, and affects the health of the next generation.

Aim: to evaluate the impact of Instruction booklet about woman rights in reproductive health on developing nursing students knowledge and misconcepts. A quasi-experimental research design. This study was conducted at technical institute of Nursing at Ismailia governorate Purposive sample of 200 female nursing students from 2nd academic year in previous mentioned setting were included in study sample (20192020). Tools of data collection: Structured Interviewing questionnaire and Likert scale.

Results: A statistically significant improvement was observed among studied sample knowledge and their attitude post intervention compared to pre intervention, Conclusion: it was concluded that there was a highly statistically significant improvement in nursing students' knowledge and concept after application of guideline about woman rights in reproductive health. Recommendation: the present study an Instruction booklet was suggested to be replicated in another setting and on another sampling.
\end{abstract}

Keywords: Reproductive rights; Attitude; Instruction booklet; Sexual and reproductive health and rights (SRHR)

\section{Introduction}

Reproductive health is an important component of general health and central feature of human development. It is a reflection of health during childhood, and crucial during adolescence and adulthood, sets the stage for health beyond the reproductive years for both women and men, and affects the health of the next generation. The health of newborn is largely a function of women health and nutrition status and her access to health care [1].

Sexual and reproductive health and rights (SRHR) encompass the right of all individuals to make decisions about sexual activity and reproduction free from discrimination, coercion and violence, and to achieve the highest attainable standard of sexual health.
Access to sexual and reproductive health and rights services allows individuals to choose whether, when and with whom to engage in sexual activity; to choose whether and when to have children; and to have access to the information and means to make those choices [2]. Additionally, reproductive rights embrace certain human rights that are already recognized in national laws, international human rights documents. It also includes the right of all to make decisions concerning reproduction free of discrimination, coercion and violence as expressed in human rights documents. In the exercise of this right, they should take into account the needs of their living and future children and their responsibilities towards the community [3]. 
Moreover, Reproductive and sexual rights were first officially recognized at the International Conference on Population and Development in Cairo in 1994. The Program of Action of International Conference on Population and Development recognized that meeting the reproductive health needs is a vital requirement for human and social development. Protecting and promoting the reproductive and sexual rights of the youth and empowering them to make informed choices is a key to wellbeing [4]. Nurses plays an important role for reproductive health rights in a variety of settings, including communities, schools, and public health and acute care clinics, which affords them many opportunities to improve adolescents' sexual and reproductive health and reduce the rates of unplanned pregnancy and sexually transmitted infections. To ensure that adolescents have access to sexual and reproductive health care which includes both preventive counseling and treatment in all nursing practice sites, nurses need to gain the knowledge and hone the skills required to deliver evidence-based counseling and services to adolescents and parents [5].

\section{Aim}

To evaluate the impact of Instruction booklet about woman rights in reproductive health on developing nursing students knowledge and Misconcepts.

\section{Methods}

A Quasi experimental design. This study was conducted at health technical institute of Nursing at Ismailia governorate. On all female nursing student were selected purposively from 2 nd academic year in previous mentioned setting during (2019- 2020 academic year ), and was eligible included in the study, their age (19-20 years).The female nursing student with SRHR included in pretest $(n=200)$ while female nursing student who have specific health education in the posttest $(n=200)$. They sample. Pilot study: The pilot study was conducted on $10 \%$ of the total sample (20 students), to test the applicability and clarity of the tools. Pilot study was excluded from study sample.

\section{Data collection tools}

Two tools were used: Tool (I) Structured interviewing questionnaire schedule sheet. It was designed in an Arabic language in forms of close and open ended questions, it composed of two parts:

Part (1): socio- demographic characteristics: such as age, marital status, religious...etc.).

Part (2): assessment of knowledge about reproductive health and rights, it consisted of eight items:-

a) Female genital mutilation, it consisted of three items.

b) Marriage and premarital counseling, it consisted of three items.

c) Breast feeding, it included three items such as (definition of breast feeding, benefits of breast feeding for mother and benefits of breast feeding for child)

d) Family planning it included three items such as (definition of family planning, types of family planning methods... ect).

e) Sexually transmitted disease it included three items (concept of sexually transmitted disease, methods of transmission of sexually transmitted disease, ...etc.).

f) Reproductive rights it included fifteen items (concept of reproductive rights and components of reproductive rights such as woman right to privacy, woman right to life,...... etc.)

g) Each item was scored as 2 for correct knowledge and 1 for incorrect knowledge

h) Scoring system:

i) Satisfactory knowledge when total knowledge scores more than $60 \%$.

j) Unsatisfactory knowledge when total knowledge scores less than $60 \%$

\section{Validity and reliability}

The version of tools was done by a panel of three expertise of professors of the obstetrics and women health at faculty of nursing to measure the validity of tools.

Field work: To fulfill the aim of the study, the following phases were adopted. Interviewing and assessment phase, designing phase, implementation phase and evaluation phase. These phases were carried out at the beginning of first semester of the academic year from beginning of October 2019 to end of December 2019 covered three months. The researcher attended three days/week, (Sunday, Tuesday\& Thursday) from 9.00Am to $12 \mathrm{pm}$. Interviewing study sample to collect socio-demographic characteristics, baseline data about students' knowledge regarding reproductive health and rights through asking questions about reproductive health and rights. At the beginning of interview the researcher greeted the students, introduced herself to each group of students included in the study, explained the purpose of the study and provided the student with all information about the study (purpose, duration, and activities) and take oral consent. Then15 student was interviewed and their knowledge and misconcepts was assessed through different tools as pretest. Data were collected by the researcher through administration of the tools to each student.

Designing phase: Based on results obtained from pre intervention assessment phase of study sample that contains students' knowledge and misconcepts regarding reproductive health and rights. The guideline was developed, sessions number and its contents, different methods of teaching, and instructional media were determined accordingly to study sample.

Implementation phase: The researcher began with implementation of pretest to identify students' knowledge and 


\section{Journal of Gynecology and Women's Health}

misconcept about reproductive health and rights. The students were divided into small groups (total number of groups were 10 groups) each group consists of 20 students. Eight sessions were implemented for each group separately. The students obtain the basic knowledge and correct their misconcept about reproductive health and rights. At the beginning of first session an orientation to the guideline and purpose, duration of each session 45 minute .Starting time was determined according to student's suitable time. At the beginning of the first session the student were oriented with the guideline contents. Each group of students was informed about the time of the next sessions at the end of the session.

The subsequent session started by feedback about the previous session and the objectives of the new session. Different methods of teaching were used such as discussion, demonstration. Instructional media included all content of educational booklet regarding reproductive health and rights which constructed by the researchers in a simple Arabic language after reviewing the related literatures. At the end of each session, student's questions were discussed to correct any misunderstanding. The total time for all sessions for each group for about six hours and the total time for all sessions for all groups for about sixteen hours.

Ethical Considerations : Participants were informed of the voluntary nature or study and the aim was explained. Students were informed about their rights to refuse or withdraw at any time without giving reason and with no untoward consequences .the study maneuver could not entail any harmful effects on participants.

Administrative design: The necessary official permissions for data collection were obtained from the dean of health technical institute of nursing. The title and objectives of study were illustrated as well as the main data item to be covered.

Statistical design: Data was verified prior to computerized entry. The Statistical Package for Social Sciences (SPSS version 20.0) was used for that purpose, followed by data tabulation and analysis. Descriptive statistics were applied (e.g., mean, standard deviation, frequency and percentages). Test of significance (chisquare, fisher exact test), Pearson correlation coefficients were used. A significant level value was considered when $p \leq 0.05$. And a highly significant level value was considered when $\mathrm{p}<0.001$.

Result

Table 1: Distribution of the studied sample according to total level of knowledge regarding reproductive health and rights $(n=200)$.

\begin{tabular}{|c|c|c|c|c|c|c|c|c|c|c|c|c|c|c|}
\hline Phases & \multicolumn{4}{|c|}{ Pre intervention $n=200$} & \multicolumn{4}{|c|}{$\begin{array}{l}\text { Immediate post intervention } \\
\qquad \mathrm{n}=\mathbf{2 0 0}\end{array}$} & \multirow{3}{*}{$\begin{array}{l}X_{1}^{2} / / \text { FET } \\
\text { (P1 value) }\end{array}$} & \multicolumn{4}{|c|}{$\begin{array}{l}\text { after } 2 \text { months' post intervention } \\
\qquad n=200\end{array}$} & \multirow{3}{*}{$\begin{array}{l}X_{1}^{2} / / \text { FET } \\
\text { (P1 value) }\end{array}$} \\
\hline Items of knowledge & \multicolumn{2}{|c|}{$\begin{array}{c}\text { Correct } \\
\text { knowledge }\end{array}$} & \multicolumn{2}{|c|}{$\begin{array}{c}\text { Incorrect } \\
\text { knowledge }\end{array}$} & \multicolumn{2}{|c|}{$\begin{array}{c}\text { Correct } \\
\text { knowledge }\end{array}$} & \multicolumn{2}{|c|}{$\begin{array}{c}\text { Incorrect } \\
\text { knowledge }\end{array}$} & & \multicolumn{2}{|c|}{$\begin{array}{l}\text { Correct knowl- } \\
\text { edge }\end{array}$} & \multicolumn{2}{|c|}{$\begin{array}{l}\text { Incorrect knowl- } \\
\text { edge }\end{array}$} & \\
\hline Items of knowledge & No & $\%$ & No & $\%$ & No & $\%$ & No & $\%$ & & No & $\%$ & No & $\%$ & \\
\hline \multirow{2}{*}{ Reproductive healths } & \multirow{2}{*}{56} & \multirow{2}{*}{28} & \multirow{2}{*}{144} & \multirow[b]{2}{*}{72} & \multirow[b]{2}{*}{200} & \multirow{2}{*}{100} & \multirow[b]{2}{*}{0} & \multirow{2}{*}{0} & 169.35 & \multirow[b]{2}{*}{184} & \multirow[b]{2}{*}{92} & \multirow[b]{2}{*}{16} & \multirow[b]{2}{*}{8} & 130.951 \\
\hline & & & & & & & & & $0.000^{* *}$ & & & & & $0.000^{* *}$ \\
\hline \multirow{2}{*}{$\begin{array}{l}\text { Reproductive health } \\
\text { facilities }\end{array}$} & \multirow{2}{*}{79} & \multirow{2}{*}{39.5} & \multirow{2}{*}{121} & \multirow{2}{*}{60.5} & \multirow{2}{*}{168} & \multirow{2}{*}{84} & \multirow{2}{*}{32} & & 62.9 & & & & & 45.943 \\
\hline & & & & & & & & 16 & $0.000^{* *}$ & 156 & 78 & 44 & 22 & $0.000^{* *}$ \\
\hline Female genital mu- & 44 & 22 & 156 & 78 & 178 & 89 & 22 & 11 & 135.281 & 164 & 82 & 36 & 18 & 106.244 \\
\hline & & & & & & & & & $0.000^{* *}$ & & & & & $0.000^{* *}$ \\
\hline premarital examina- & (2) & a & (2) & r & 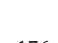 & (0) & (2) & (s) & 59.1885 & (est & 然 & (2) & se & 38.185 \\
\hline tion \&counseling & 94 & 47 & 106 & 53 & 176 & 88 & 24 & 12 & $0.000^{* *}$ & 164 & 82 & 36 & 18 & $0.000^{* *}$ \\
\hline Methods of family & 100 & 50 & 100 & 50.0 & 196 & 98 & 4 & 2 & 95.272 & 188 & 94 & 12 & 6 & 75.955 \\
\hline & & & & & & & & & $0.000^{* *}$ & & & & & $0.000^{* *}$ \\
\hline Democt fordin & 122 & 6 & 60 & 2 & 102 & 06 & 0 & 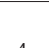 & 45.958 & 102 & 06 & 0 & 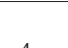 & 44.16 \\
\hline Breast feeding & 132 & 66 & 68 & 34 & 192 & 96 & 8 & 4 & $0.000^{* *}$ & 192 & 96 & 8 & 4 & $0.000^{* *}$ \\
\hline Sexually transmitted & 1 & 2 & 156 & 70 & 176 & 00 & 2 & 12 & 59.185 & $10^{-1}+2$ & & 26 & & 70.882 \\
\hline diseases & 44 & 22 & 156 & 78 & 176 & 88 & 24 & 12 & $0.000^{* *}$ & 164 & 82 & 36 & 18 & $0.000^{* *}$ \\
\hline rights of woman in & 79 & 395 & 121 & 605 & 200 & 100 & 0 & 0 & 135.183 & 100 & 00 & 2 & 1 & 130.93 \\
\hline reproductive health & & & 121 & 60.5 & 200 & 100 & 0 & 0 & $0.000^{* *}$ & 198 & 99 & 2 & 1 & $0.000^{* *}$ \\
\hline
\end{tabular}

A statistical significant difference $(\mathrm{P} \leq 0.05)$

$X_{1}^{2}\left(\mathrm{P}_{1}\right.$ value $)=$ Pre and post intervention

A highly statistical significant difference $(P \leq 0.001)$

$X_{2}^{2}\left(\mathrm{P}_{2}-\right.$ value $)=$ Pre and after 4 weeks

FET: Fisher Exact Test 
(Table 1): revealed that, the studied sample had incorrect knowledge about reproductive health and rights pre intervention and also had correct total knowledge immediate post intervention and 8 weeks post intervention after implementation of Instruction booklet and there was highly statistically significant improvement of total knowledge score post intervention after implementation of Instruction booklet ( $\mathrm{P} \leq$ 0.001). (Table 2) illustrated that, more than two thirds of studied sample had incorrect concept about female genital mutation during pre-intervention and also had highly statistically significant improvement of their concept immediate after implementation of Instruction booklet and after 2 months' post intervention compared to pre intervention $(\mathrm{P} \leq$ $0.001)$.

Table 2: Distribution of the studied sample according to misconception about female genital mutation $(n=200)$.

\begin{tabular}{|c|c|c|c|c|c|c|c|c|c|c|c|c|c|c|}
\hline \multirow{3}{*}{$\begin{array}{c}\text { Phases } \\
\text { Items }\end{array}$} & \multicolumn{4}{|c|}{ Pre intervention $n=200$} & \multicolumn{4}{|c|}{$\begin{array}{c}\text { Immediate post } \\
\text { intervention } \\
n=200\end{array}$} & \multirow{3}{*}{ (P1 value) } & \multicolumn{4}{|c|}{$\begin{array}{l}\text { after } 2 \text { months' post intervention } \\
\qquad n=200\end{array}$} & \multirow{3}{*}{$X_{2}^{2}$ (P2 - value) } \\
\hline & \multicolumn{2}{|c|}{ Yes } & \multicolumn{2}{|c|}{ No } & \multicolumn{2}{|c|}{ Yes } & \multicolumn{2}{|c|}{ No } & & Yes & & No & & \\
\hline & No. & $\%$ & No. & $\%$ & No. & $\%$ & No. & $\%$ & & No. & $\%$ & No. & $\%$ & \\
\hline $\begin{array}{l}\text { Female genital } \\
\text { mutation is } \\
\text { important for } \\
\text { girl future life }\end{array}$ & 142 & 71 & 58 & 29 & 28 & 14 & 172 & 86 & $\begin{array}{l}100.781 \\
0.000^{* *}\end{array}$ & 36 & 18 & 164 & 82 & $\begin{array}{l}86.315 \\
0.000^{* *}\end{array}$ \\
\hline $\begin{array}{l}\text { Female genital } \\
\text { mutation pre- } \\
\text { vent inflamma- } \\
\text { tion \& cancer of } \\
\text { female genital } \\
\text { tract }\end{array}$ & 120 & 60 & 80 & 40 & 32 & 16 & 168 & 84 & $\begin{array}{c}61.226 \\
0.000^{* *}\end{array}$ & 30 & 15 & 170 & 85 & $\begin{array}{l}65.422 \\
0.000^{* *}\end{array}$ \\
\hline $\begin{array}{l}\text { Female genital } \\
\text { mutation not } \\
\text { affect female } \\
\text { attitude }\end{array}$ & 112 & 56 & 88 & 44 & 46 & 23 & 154 & 77 & $\begin{array}{c}34.722 \\
0.000^{* *}\end{array}$ & 40 & 20 & 160 & 80 & $\begin{array}{l}40.986 \\
0.000^{* *}\end{array}$ \\
\hline
\end{tabular}

$X_{1}^{2}\left(\mathrm{P}_{1}\right.$ value $)=$ Pre and post intervention

$X_{2}^{2}\left(\mathrm{P}_{2}-\right.$ value $)=$ Pre and after 4 weeks

${ }^{* \star} A$ highly statistical significant difference $(P \leq 0.001)$

(Table 3) shows that, more than half of studied sample had incorrect concept about menstruation during pre-intervention and also had highly statistically significant improvement of concepts about menstruation immediate after implementation of Instruction booklet and after 2 months' post intervention compared to pre intervention $(\mathrm{P} \leq 0.001)$. (Table 4) demonstrates that, more than half of studied sample had incorrect concept about marriage during pre-intervention and also had highly statistically significant improvement of concepts regarding to marriage immediate after implementation of Instruction booklet and after 2 months' post intervention compared to pre intervention $(\mathrm{P} \leq$ $0.001)$.

Table 3: Distribution of the studied sample according to misconception about menstruation $(n=200)$.

\begin{tabular}{|c|c|c|c|c|c|c|c|c|c|c|c|c|c|c|}
\hline \multirow{3}{*}{$\begin{array}{l}\text { Phases } \\
\text { Items }\end{array}$} & \multicolumn{4}{|c|}{$\begin{array}{l}\text { Pre intervention } \\
\quad n=200\end{array}$} & \multicolumn{4}{|c|}{$\begin{array}{l}\text { Immediate post intervention } \\
\qquad n=200\end{array}$} & \multirow{3}{*}{$X_{1}^{2}$ (P1 value) } & \multicolumn{4}{|c|}{$\begin{array}{l}\text { after } 2 \text { months' post inter- } \\
\text { vention } n=200\end{array}$} & \multirow{3}{*}{$X_{2}^{2}$ (P2 - value $)$} \\
\hline & \multicolumn{2}{|c|}{ Yes } & \multicolumn{2}{|c|}{ No } & \multicolumn{2}{|c|}{ Yes } & \multicolumn{2}{|c|}{ No } & & \multicolumn{2}{|c|}{ Yes } & \multicolumn{2}{|c|}{ No } & \\
\hline & No. & $\%$ & No. & $\%$ & No. & $\%$ & No. & $\%$ & & No. & $\%$ & No. & $\%$ & \\
\hline \multirow{2}{*}{$\begin{array}{l}\text { Avoid taking } \\
\text { shower during } \\
\text { menstruation }\end{array}$} & \multirow{2}{*}{86} & \multirow[b]{2}{*}{43} & \multirow{2}{*}{114} & \multirow[b]{2}{*}{57} & \multirow[b]{2}{*}{26} & \multirow[b]{2}{*}{13} & \multirow[b]{2}{*}{174} & \multirow[b]{2}{*}{87} & 33.729 & \multirow{2}{*}{20} & \multirow{2}{*}{10} & \multirow{2}{*}{180} & \multirow{2}{*}{90} & 41.257 \\
\hline & & & & & & & & & $0.000^{* *}$ & & & & & $0.000^{* *}$ \\
\hline \multirow{2}{*}{$\begin{array}{l}\text { Taking analgesic } \\
\text { during men- } \\
\text { struation affect } \\
\text { female fertility }\end{array}$} & \multirow[b]{2}{*}{120} & \multirow[b]{2}{*}{60} & \multirow[b]{2}{*}{80} & \multirow[b]{2}{*}{40} & \multirow[b]{2}{*}{28} & \multirow[b]{2}{*}{14} & \multirow[b]{2}{*}{172} & \multirow[b]{2}{*}{86} & 67.593 & \multirow[b]{2}{*}{26} & \multirow[b]{2}{*}{13} & \multirow[b]{2}{*}{174} & & 72.086 \\
\hline & & & & & & & & & $0.000^{* *}$ & & & & 87 & $0.000^{* *}$ \\
\hline Avoid cutting & & & & & & & & & 31.362 & & & & & 37.48 \\
\hline $\begin{array}{l}\text { hair during } \\
\text { menstruation }\end{array}$ & 100 & 50 & 100 & 50 & 44 & 22 & 156 & 78 & $0.000^{* *}$ & 38 & 19 & 162 & 81 & $0.000^{* *}$ \\
\hline
\end{tabular}




\section{Journal of Gynecology and Women's Health}

\begin{tabular}{|c|c|c|c|c|c|c|c|c|c|c|c|c|c|c|}
\hline \multirow{2}{*}{$\begin{array}{l}\text { Avoid cutting } \\
\text { nail during } \\
\text { menstruation }\end{array}$} & \multirow[b]{2}{*}{116} & \multirow[b]{2}{*}{58} & \multirow[b]{2}{*}{84} & \multirow[b]{2}{*}{42} & \multirow[b]{2}{*}{68} & \multirow[b]{2}{*}{34} & \multirow[b]{2}{*}{132} & \multirow[b]{2}{*}{66} & 17.391 & \multirow[b]{2}{*}{56} & \multirow[b]{2}{*}{28} & \multirow[b]{2}{*}{144} & \multirow[b]{2}{*}{72} & 26.281 \\
\hline & & & & & & & & & $0.000^{* *}$ & & & & & $0.000^{* *}$ \\
\hline \multirow{2}{*}{$\begin{array}{l}\text { Not important } \\
\text { to seek medical } \\
\text { advice }\end{array}$} & \multirow{2}{*}{140} & \multirow{2}{*}{70} & \multirow{2}{*}{60} & \multirow{2}{*}{30} & \multirow{2}{*}{38} & \multirow{2}{*}{19} & \multirow{2}{*}{162} & \multirow{2}{*}{81} & 77.9 & \multirow{2}{*}{34} & \multirow{2}{*}{17} & \multirow{2}{*}{166} & \multirow{2}{*}{83} & 84.57 \\
\hline & & & & & & & & & $0.000^{* *}$ & & & & & $0.000^{* *}$ \\
\hline
\end{tabular}

$X_{1}^{2}\left(\mathrm{P}_{1}\right.$ value $)=$ Pre and post intervention

$X_{2}^{2}\left(\mathrm{P}_{2}-\right.$ value $)=$ Pre and after 3 weeks

${ }^{* *} A$ highly statistical significant difference $(P \leq 0.001)$

Table 4: Distribution of the studied sample according to misconception about marriage $(n=200)$.

\begin{tabular}{|c|c|c|c|c|c|c|c|c|c|c|c|c|c|c|}
\hline Phases & \multicolumn{4}{|c|}{$\begin{array}{l}\text { Pre intervention } \\
\quad \mathbf{n}=200\end{array}$} & \multicolumn{4}{|c|}{$\begin{array}{l}\text { Immediate post intervention } \\
\qquad \mathrm{n}=200\end{array}$} & \multirow{3}{*}{$X_{1}^{2}$ (P1 value) } & \multicolumn{4}{|c|}{$\begin{array}{l}\text { after } 2 \text { months' post } \\
\text { intervention } n=200\end{array}$} & \multirow{3}{*}{$X_{2}^{2}$ (P2-value $)$} \\
\hline \multirow{2}{*}{ Items } & \multicolumn{2}{|c|}{ Yes } & \multicolumn{2}{|c|}{ No } & \multicolumn{2}{|c|}{ Yes } & \multicolumn{2}{|c|}{ No } & & \multicolumn{2}{|c|}{ Yes } & \multicolumn{2}{|c|}{ No } & \\
\hline & No. & $\%$ & No. & $\%$ & No. & $\%$ & No. & $\%$ & & No. & $\%$ & No. & $\%$ & \\
\hline \multirow{2}{*}{$\begin{array}{l}\text { Advice for early } \\
\text { marriage before } \\
\text { twenty }\end{array}$} & \multirow[b]{2}{*}{100} & \multirow[b]{2}{*}{50} & \multirow[b]{2}{*}{100} & \multirow[b]{2}{*}{50} & \multirow[b]{2}{*}{36} & \multirow[b]{2}{*}{18} & \multirow[b]{2}{*}{164} & \multirow[b]{2}{*}{82} & 35.994 & \multirow[b]{2}{*}{30} & \multirow[b]{2}{*}{15} & \multirow[b]{2}{*}{170} & \multirow[b]{2}{*}{85} & 46.105 \\
\hline & & & & & & & & & $0.000^{* *}$ & & & & & $0.000^{* *}$ \\
\hline \multirow{2}{*}{$\begin{array}{l}\text { Advice for relative } \\
\text { marriage }\end{array}$} & \multirow{2}{*}{106} & \multirow{2}{*}{53} & \multirow{2}{*}{94} & \multirow{2}{*}{47} & \multirow{2}{*}{50} & \multirow{2}{*}{25} & \multirow{2}{*}{150} & \multirow{2}{*}{75} & 25.073 & \multirow{2}{*}{32} & \multirow{2}{*}{16} & \multirow{2}{*}{168} & 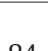 & 45.624 \\
\hline & & & & & & & & & $0.000^{* *}$ & & & & 84 & $0.000^{* *}$ \\
\hline Premarital & & & & & & & & & 11.946 & & & & & 16.897 \\
\hline $\begin{array}{l}\text { counseling not } \\
\text { important }\end{array}$ & 80 & 40 & 120 & 60 & 44 & 22 & 156 & 78 & $0.000^{* *}$ & 38 & 19 & 162 & 81 & $0.000^{* *}$ \\
\hline
\end{tabular}

$X_{1}^{2}\left(\mathrm{P}_{1}\right.$ value $)=$ Pre and post intervention

$X_{2}^{2}\left(P_{2}-\right.$ value $)=$ Pre and after 3 weeks

${ }^{* *} A$ highly statistical significant difference $(P \leq 0.001)$

(Table 5) clarified that, there was highly statistical significant improvement of their concept about pregnancy immediate after implementation of Instruction booklet and after 2 months' post intervention compared to pre intervention ( $\mathrm{P} \leq 0.001$ ). (Table 6 ) indicated that, less than half of studied sample had incorrect

concept about labor during pre-intervention and also had highly statistically significant improvement of concepts about labor immediate after implementation of Instruction booklet and after 2 months' post intervention compared to pre intervention (P $\leq$ $0.001)$.

Table 5: Distribution of the studied sample according to misconception about pregnancy ( $n=200)$.

\begin{tabular}{|c|c|c|c|c|c|c|c|c|c|c|c|c|c|c|}
\hline Phases & \multicolumn{4}{|c|}{ Pre intervention $\mathbf{n}=\mathbf{2 0 0}$} & \multicolumn{4}{|c|}{$\begin{array}{l}\text { Immediate post intervention } \\
\qquad \mathrm{n}=\mathbf{2 0 0}\end{array}$} & \multirow{3}{*}{ (P1 value) } & \multicolumn{4}{|c|}{$\begin{array}{l}\text { after } 2 \text { months' post } \\
\text { intervention } n=200\end{array}$} & \multirow{3}{*}{ (P2-Value) } \\
\hline \multirow{2}{*}{ Items } & \multicolumn{2}{|c|}{ Yes } & \multicolumn{2}{|c|}{ No } & \multicolumn{2}{|c|}{ Yes } & \multicolumn{2}{|c|}{ No } & & \multicolumn{2}{|c|}{ Yes } & \multicolumn{2}{|c|}{ No } & \\
\hline & No. & $\%$ & No. & $\%$ & No. & $\%$ & No. & $\%$ & & No. & $\%$ & No. & $\%$ & \\
\hline \multirow{2}{*}{$\begin{array}{l}\text { Delayed pregnancy } \\
\text { consider infertility }\end{array}$} & \multirow{2}{*}{78} & \multirow{2}{*}{39} & \multirow{2}{*}{122} & \multirow{2}{*}{61} & \multirow{2}{*}{56} & \multirow{2}{*}{28} & \multirow{2}{*}{144} & \multirow{2}{*}{72} & 4.103 & \multirow{2}{*}{50} & \multirow{2}{*}{25} & \multirow{2}{*}{150} & \multirow{2}{*}{75} & 7.849 \\
\hline & & & & & & & & & $0.051^{*}$ & & & & & $0.004^{* *}$ \\
\hline \multirow{2}{*}{$\begin{array}{l}\text { Not important to } \\
\text { follow up pregnancy } \\
\text { after cessation of } \\
\text { menstruation }\end{array}$} & \multirow[t]{2}{*}{146} & \multirow[t]{2}{*}{73} & \multirow[t]{2}{*}{54} & \multirow[t]{2}{*}{27} & \multirow[t]{2}{*}{44} & \multirow[t]{2}{*}{22} & \multirow[t]{2}{*}{156} & \multirow[t]{2}{*}{78} & 76.933 & \multirow[t]{2}{*}{40} & \multirow[t]{2}{*}{20} & 160 & 80 & 82.182 \\
\hline & & & & & & & & & $0.000^{* *}$ & & & & & $0.000^{* *}$ \\
\hline Recommended & & & & & & & & & 28.832 & & & & & 37.006 \\
\hline $\begin{array}{l}\text { vaccination not } \\
\text { important }\end{array}$ & 112 & 56 & 88 & 44 & 50 & 25 & 150 & 75 & $0.000^{* *}$ & 40 & 20 & 160 & 80 & $0.000^{* *}$ \\
\hline don't taking cnace & & & & & & & & & 4.812 & & & & & 8.919 \\
\hline $\begin{array}{l}\text { between pregnan- } \\
\text { cies }\end{array}$ & 60 & 30 & 140 & 70 & 36 & 18 & 164 & 82 & $0.016^{*}$ & 32 & 16 & 168 & 84 & $0.003^{* *}$ \\
\hline
\end{tabular}

$X_{1}^{2} \quad\left(\mathrm{P}_{1}\right.$ value $)=$ Pre and immediate post intervention

$X_{2}^{2}\left(\mathrm{P}_{2}-\right.$ value $)=$ Pre and after 3 weeks

${ }^{*}$ A statistical significant difference $(P \leq 0.05)$

${ }^{* *} \mathrm{~A}$ highly statistical significant difference $(\mathrm{P} \leq 0.001)$ 


\section{Journal of Gynecology and Women's Health}

Table 6: Distribution of the studied sample according to misconception about labor $(n=150)$.

\begin{tabular}{|c|c|c|c|c|c|c|c|c|c|c|c|c|c|c|}
\hline Phases & \multicolumn{4}{|c|}{$\begin{array}{l}\text { Pre intervention } \\
\quad \mathbf{n}=200\end{array}$} & \multicolumn{4}{|c|}{$\begin{array}{l}\text { Immediate post intervention } \\
\qquad \mathrm{n}=\mathbf{2 0 0}\end{array}$} & \multirow{3}{*}{$X_{1}^{2}$ (P1-Value) } & \multicolumn{4}{|c|}{$\begin{array}{l}\text { After } 3 \text { weeks post } \\
\text { intervention } n=200\end{array}$} & \multirow{3}{*}{$X_{2}^{2}$ (P2-Value) } \\
\hline \multirow{2}{*}{ Items } & Yes & & No & & Yes & & No & & & \multicolumn{2}{|c|}{ Yes } & \multicolumn{2}{|c|}{ No } & \\
\hline & No. & $\%$ & No. & $\%$ & No. & $\%$ & No. & $\%$ & & No & $\%$ & No. & $\%$ & \\
\hline \multirow{2}{*}{$\begin{array}{l}\text { Home } \\
\text { delivery is } \\
\text { best than } \\
\text { hospital } \\
\text { delivery }\end{array}$} & \multirow[b]{2}{*}{96} & \multirow[b]{2}{*}{48} & \multirow[b]{2}{*}{104} & \multirow[b]{2}{*}{52} & \multirow[b]{2}{*}{36} & \multirow[b]{2}{*}{18} & \multirow[b]{2}{*}{164} & \multirow[b]{2}{*}{82} & 29.929 & \multirow[b]{2}{*}{30} & \multirow[b]{2}{*}{15} & \multirow[b]{2}{*}{170} & \multirow[b]{2}{*}{85} & 37.1986 \\
\hline & & & & & & & & & $0.000^{* *}$ & & & & & $0.000^{* *}$ \\
\hline \multirow{2}{*}{$\begin{array}{l}\text { Perineal } \\
\text { care after } \\
\text { delivery } \\
\text { start down } \\
\text { ward to } \\
\text { upward. }\end{array}$} & \multirow[b]{2}{*}{86} & \multirow[b]{2}{*}{43} & \multirow[b]{2}{*}{114} & \multirow[b]{2}{*}{57} & \multirow[b]{2}{*}{60} & \multirow[b]{2}{*}{30} & \multirow[b]{2}{*}{140} & \multirow[b]{2}{*}{70} & 6.187 & \multirow[b]{2}{*}{54} & \multirow[b]{2}{*}{27} & \multirow[b]{2}{*}{146} & \multirow[b]{2}{*}{73} & 8.151 \\
\hline & & & & & & & & & $0.015^{*}$ & & & & & $0.005^{* *}$ \\
\hline \multirow{2}{*}{$\begin{array}{l}\text { Avoid tak- } \\
\text { ing shower } \\
\text { one week } \\
\text { after labor }\end{array}$} & \multirow[b]{2}{*}{80} & \multirow[b]{2}{*}{40} & & & & & & & 19.144 & & & & & 23.934 \\
\hline & & & 120 & 60 & 34 & 17 & 166 & 83 & $0.000^{* *}$ & 30 & 15 & 170 & 85 & $0.000^{* *}$ \\
\hline
\end{tabular}

$X_{1}^{2}\left(\mathrm{P}_{1}\right.$ value $)=$ Pre and post intervention

$X_{2}^{2}\left(\mathrm{P}_{2}-\right.$ value $)=$ Pre and after 3 weeks

${ }^{*}$ A statistical significant difference $(P \leq 0.05)$

${ }^{* *} A$ highly statistical significant difference $(P \leq 0.001)$

(Table 7) illustrated that, there were highly statistically significant improvement of misconception about breast feeding immediate after implementation of Instruction booklet and after 2 months' post intervention compared to pre intervention $(\mathrm{P} \leq$

$\%$ were had correct concept about reproductive health during immediate post intervention. Also $98.0 \%$ of them were had correct concept three weeks post intervention compared to pre intervention. 0.001) (Figure 1\&2): showed that, most of studied sample 87.0

Table 7: Distribution of the studied sample according to misconception about breast feeding $(n=200)$.

\begin{tabular}{|c|c|c|c|c|c|c|c|c|c|c|c|c|c|c|}
\hline Phases & \multicolumn{4}{|c|}{$\begin{array}{l}\text { Pre intervention } \\
\quad n=200\end{array}$} & \multicolumn{4}{|c|}{$\begin{array}{l}\text { Immediate post intervention } \\
\qquad n=200\end{array}$} & \multirow{3}{*}{$X_{1}^{2}$ (P1 value) } & \multicolumn{4}{|c|}{$\begin{array}{l}\text { after } 2 \text { months' post } \\
\text { intervention } n=200\end{array}$} & \multirow{3}{*}{$X_{2}^{2}$ (P2 - value $)$} \\
\hline \multirow{2}{*}{ Items } & \multicolumn{2}{|c|}{ Yes } & \multicolumn{2}{|c|}{ No } & \multicolumn{2}{|c|}{ Yes } & \multicolumn{2}{|c|}{ No } & & \multicolumn{2}{|c|}{ Yes } & \multicolumn{2}{|c|}{ No } & \\
\hline & No. & $\%$ & No. & $\%$ & No. & $\%$ & No. & $\%$ & & No. & $\%$ & No. & $\%$ & \\
\hline \multirow{2}{*}{$\begin{array}{l}\text { Breast feeding not } \\
\quad \text { important }\end{array}$} & \multirow{2}{*}{88} & \multirow{2}{*}{44} & \multirow{2}{*}{112} & \multirow{2}{*}{56} & \multirow{2}{*}{44} & \multirow{2}{*}{22} & \multirow{2}{*}{156} & \multirow{2}{*}{78} & 14.96 & \multirow{2}{*}{40} & \multirow{2}{*}{20} & \multirow{2}{*}{160} & \multirow{2}{*}{80} & 20.1853 \\
\hline & & & & & & & & & $0.000^{* *}$ & & & & & $0.000^{* *}$ \\
\hline \multirow[b]{2}{*}{$\begin{array}{l}\text { Breast feeding not } \\
\text { decrease weight }\end{array}$} & \multirow[b]{2}{*}{68} & \multirow[b]{2}{*}{34} & \multirow[b]{2}{*}{132} & \multirow[b]{2}{*}{66} & \multirow[b]{2}{*}{44} & \multirow[b]{2}{*}{22} & \multirow[b]{2}{*}{156} & \multirow[b]{2}{*}{78} & 6.357 & \multirow[b]{2}{*}{40} & \multirow[b]{2}{*}{20} & \multirow[b]{2}{*}{160} & & 7.95 \\
\hline & & & & & & & & & $0.021^{*}$ & & & & 80 & $0.004^{* *}$ \\
\hline breast feeding & & & & & & & & & 97.154 & & & & & 119.836 \\
\hline $\begin{array}{l}\text { used as contra- } \\
\text { ceptive }\end{array}$ & 164 & 82 & 36 & 18 & 38 & 25.3 & 112 & 74.7 & $0.000^{* *}$ & 44 & 22 & 156 & 78 & $0.000^{* *}$ \\
\hline
\end{tabular}

$X_{1}^{2}\left(P_{1}\right.$ value $)=$ Pre and post intervention

$X_{2}^{2}\left(P_{2}-\right.$ value $)=$ Pre and after 3 weeks

${ }^{*}$ A statistical significant difference $(P \leq 0.05)$

${ }^{* *} A$ highly statistical significant difference $(P \leq 0.001)$

\section{Discussion}

Health systems and individuals can take a number of actions to safeguard reproductive health. These actions differ from many other health interventions in that the motivation for their use is not necessarily limited to better health and involves cultural and societal norms. Irrespective of these additional considerations, these interventions have important health implications that affect the widespread implementation and use of the services [6]. 
Providing adolescents with the means to attain high standards of health, in ways that ensure equality, nondiscrimination, privacy, and confidentiality, is an integral part of respecting and protecting globally accepted human rights. Ensuring that adolescents have access to sexual and reproductive health services requires extending the availability, accessibility, acceptability, and quality of the information and the services. Helping adolescents make a healthy transition to adulthood involves programs to protect them from unintended pregnancy, sexually transmitted infections
(STIs), and poor reproductive health outcomes [7]. The current study aimed to assess the knowledge and concepts of adolescent females regarding reproductive health rights by assessing the level of knowledge of adolescent females about reproductive health, detecting their needs, designing and implementing a Instruction booklet about women reproductive health rights according of adolescent females needs, evaluating the Impact of Instruction booklet about Woman Rights in Reproductive Health on developing Nursing Students Knowledge and Misconcept.

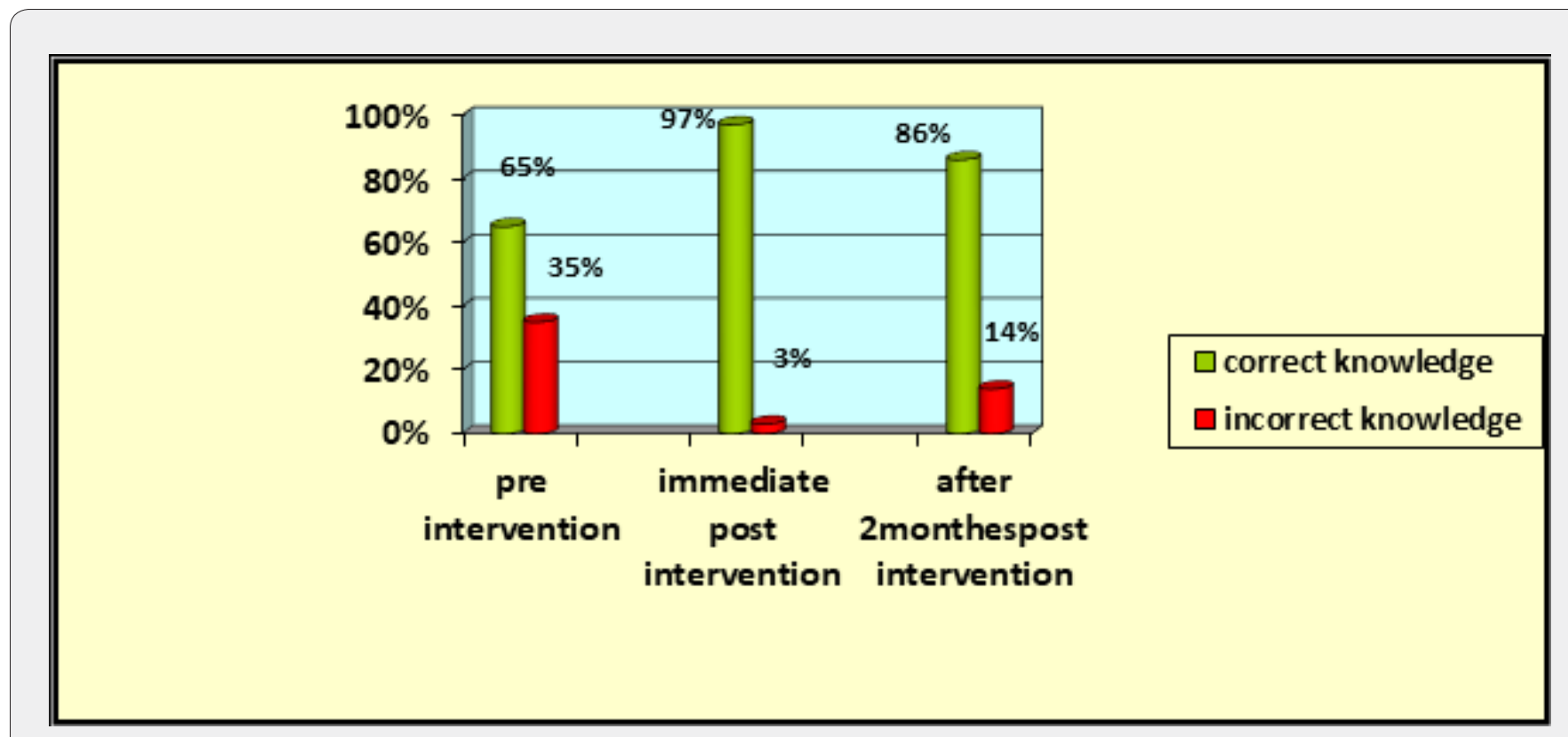

Figure 1: Distribution of the studied sample according to total level of knowledge regarding reproductive health rights $(n=200)$.

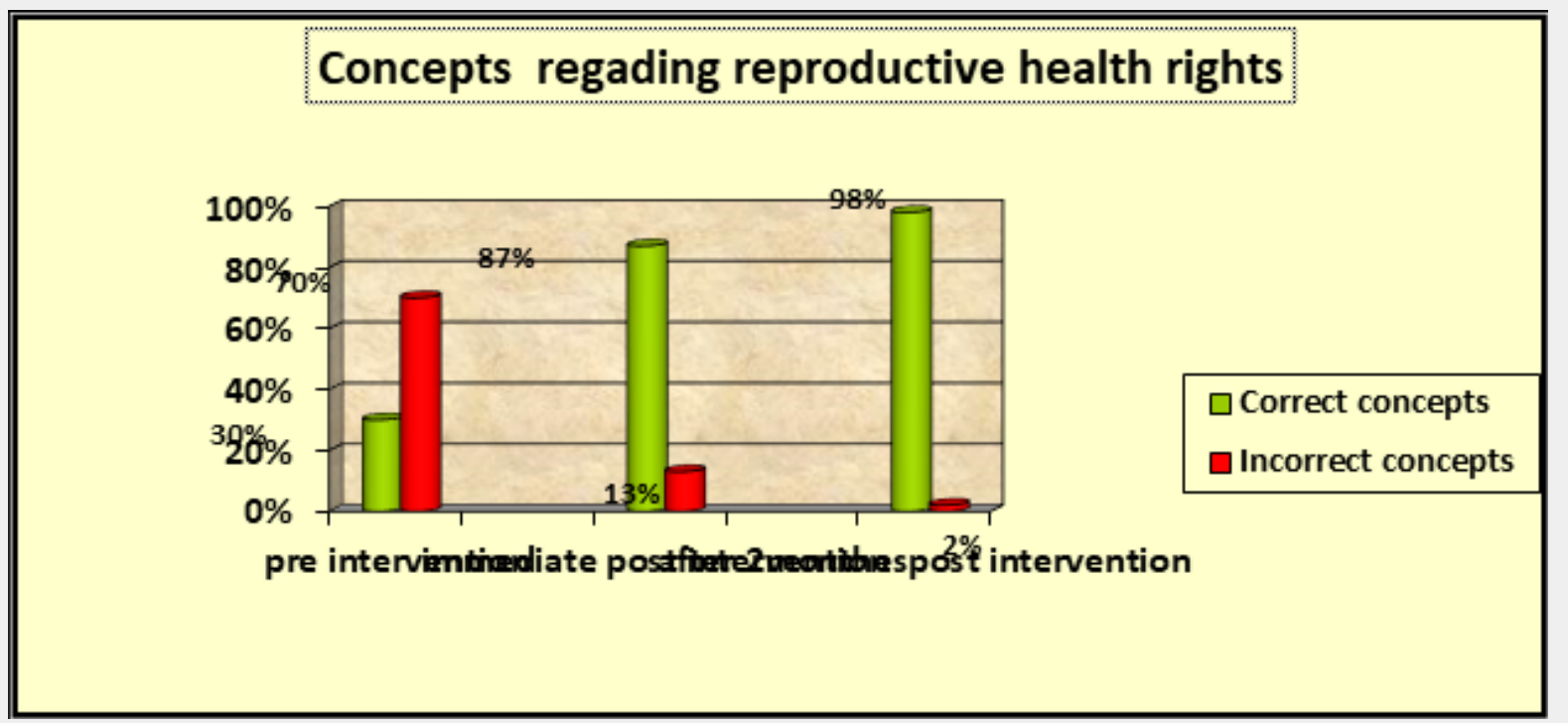

Figure 2: Distribution of studied sample according to misconception regarding reproductive health rights during different phases of assessment $(n=200)$. 
Table 8: Relation between total of knowledge level regarding reproductive health and rights and socio-demographic characteristics throughout different phases of the study $(n=200)$.

\begin{tabular}{|c|c|c|c|c|c|c|c|c|c|c|c|c|c|c|c|c|c|c|}
\hline \multirow{3}{*}{ Variable } & \multicolumn{4}{|c|}{ Pre intervention } & \multirow{3}{*}{$\begin{array}{l}\mathrm{X} 2 / \\
\text { FET }\end{array}$} & \multirow{3}{*}{ P Value } & \multirow{2}{*}{\multicolumn{2}{|c|}{$\begin{array}{c}\text { Immediate } \\
\text { post inter- } \\
\text { vention }\end{array}$}} & & & \multirow{3}{*}{$\begin{array}{l}\text { X2/ } \\
\text { FET }\end{array}$} & \multirow{3}{*}{$\begin{array}{c}P \\
\text { Value }\end{array}$} & \multicolumn{4}{|c|}{$\begin{array}{l}\text { after } 2 \text { months' } \\
\text { post intervention }\end{array}$} & \multirow{3}{*}{ X2/FET } & \multirow{3}{*}{ P Value } \\
\hline & \multicolumn{2}{|c|}{$\begin{array}{c}\text { Correct } \\
\text { knowl- } \\
\text { edge } \\
\text { n=70 }\end{array}$} & \multicolumn{2}{|c|}{$\begin{array}{c}\text { Incorrect } \\
\text { knowledge } \\
\mathbf{n = 1 3 0}\end{array}$} & & & & & $\begin{array}{r}\text { Incc } \\
\text { kn } \\
\text { edg }\end{array}$ & $\begin{array}{l}\text { rect } \\
w l- \\
n=6\end{array}$ & & & $\begin{array}{r}\text { Cor } \\
\text { kn } \\
\text { ed } \\
n=\end{array}$ & $\begin{array}{l}\text { ect } \\
\text { wl- } \\
\text { ge } \\
74\end{array}$ & $\begin{array}{r}\text { Inco } \\
\text { kn } \\
\text { ec } \\
\text { n }\end{array}$ & $\begin{array}{l}\text { rect } \\
\text { wl- } \\
\text { ge } \\
26\end{array}$ & & \\
\hline & No & $\%$ & No & $\%$ & & & No & $\%$ & No & $\%$ & & & No & $\%$ & No & $\%$ & & \\
\hline $\begin{array}{c}\text { Age } \\
\text { (years) }\end{array}$ & & & & & \multirow{3}{*}{1.187} & \multirow{3}{*}{0.5} & & & & & \multirow{3}{*}{0.19} & \multirow{3}{*}{0.909} & & & & & \multirow{3}{*}{5.357} & \multirow{3}{*}{0.069} \\
\hline $18-19$ & 18 & 25.7 & 26 & 20 & & & 42 & 22 & 2 & 20 & & & 31 & 18 & 10 & 40 & & \\
\hline $\begin{array}{l}20 \text { or } \\
\text { above }\end{array}$ & 52 & 74.3 & 104 & 80 & & & 152 & 88 & 4 & 80 & & & 143 & 82 & 16 & 60 & & \\
\hline $\begin{array}{l}\text { Marital } \\
\text { status of } \\
\text { student } \\
\text { mothers }\end{array}$ & & & & & \multirow[t]{3}{*}{10.922} & \multirow[t]{3}{*}{$0.001^{* *}$} & & & & & \multirow[t]{3}{*}{1.385} & \multirow[t]{3}{*}{0.239} & & & & & \multirow[t]{3}{*}{0.062} & \multirow[t]{3}{*}{0.72} \\
\hline Married & 56 & 80 & 125 & 96 & & & 178 & 92 & 2 & 20 & & & 160 & 92 & 23 & 90 & & \\
\hline Divorced & 14 & 20 & 5 & 4 & & & 16 & 8 & 4 & 80 & & & 14 & 8 & 3 & 10 & & \\
\hline \multicolumn{5}{|l|}{$\begin{array}{l}\text { Resi- } \\
\text { dence }\end{array}$} & \multirow{3}{*}{6.982} & \multirow{3}{*}{$0.007^{* *}$} & & & & & \multirow{3}{*}{6.519} & \multirow{3}{*}{$0.011^{*}$} & & & & & \multirow{3}{*}{16.624} & \multirow{3}{*}{$0.000^{* *}$} \\
\hline Urban & 54 & 77 & 69 & 53 & & & 122 & 63 & 6 & 100 & & & 118 & 68 & 5 & 20 & & \\
\hline Rural & 16 & 23 & 61 & 47 & & & 72 & 37 & 0 & 0 & & & 56 & 32 & 21 & 80 & & \\
\hline \multicolumn{17}{|l|}{$\begin{array}{l}\text { Mother' } \\
\text { educa- } \\
\text { tion }\end{array}$} & & \\
\hline $\begin{array}{l}\text { Basic ed- } \\
\text { ucation }\end{array}$ & 2 & 3 & 39 & 30 & & & 39 & 20 & 3 & 50 & & & 41 & 23.6 & 0 & 0 & & \\
\hline $\begin{array}{l}\text { Second- } \\
\text { ary edu- } \\
\text { cation }\end{array}$ & 41 & 58.5 & 86 & 66.1 & 43.509 & $0.000^{* *}$ & 122 & 63 & 3 & 50 & 2.484 & 0.289 & 127 & 73 & 0 & 0 & 121.154 & $0.000^{* *}$ \\
\hline $\begin{array}{l}\text { Univer- } \\
\text { sity or } \\
\text { above }\end{array}$ & 27 & 38.5 & 5 & 3.9 & & & 33 & 17 & 0 & 0 & & & 6 & 3.4 & 26 & 100 & & \\
\hline
\end{tabular}

*Statistical significant difference $(P \leq 0.05)$

** highly statistical significant difference $((P \leq 0.001)$

Menopause is one of the most critical stages of women's life which because of its symptoms and effects might significantly create health problems for women. Menopause is a normal physiological process in all women's life. Menopause signals the end of the reproductive years and is associated with signs of estrogen deficiency with a considerable impact on women's health-related quality of life (North American Menopause Society, 2012). Health is the fundamental right of every human being and improvement of educational methods is a public duty. Considering the limited research in this field, a programmed approach to learning is evident in various cycles of life including menopause as the most critical period of women's life. Lack of knowledge and access to relevant information is the major challenge faced by menopausal women intensified by contradictory information (Yazdkhasti et al., 2012).
The present study aimed to evaluate the effect of a designed educational program on menopausal symptoms. This aim is achieved through; assessing women's knowledge regarding menopausal symptoms,

assessing women's health practices toward menopausal symptoms, Designing, implementing and evaluating the outcome of the educational program on Menopausal symptoms. The findings of the current study will be discussed under five main parts namely: characteristics of both study and control groups, knowledge of both groups regarding menopause, practices towards menopausal symptoms, comparison between both study and control groups regarding menopausal symptoms before and three months after program.

Regarding to sociodemographic characteristics of adolescent 
females. The findings of the present study reveals the mean age group19.63 \pm 0.47 years old and single. This finding agreement with, Kaur et al. [8] who studied descriptive study to assess the knowledge regarding reproductive health care among B.Sc. nursing 1st year students at SGRD College of nursing, Amritsar, India, who found that the majority of subject were in the age group of 19- 20 years.

Also,results consistence with Govender, et al. [9] who studied Knowledge, attitudes and peer influences related to pregnancy, sexual and reproductive health among adolescents using maternal health services in Ugu, found that most of the participants (97.5\%, $\mathrm{n}=318$ ) were unemployed. Our finding disagrees with Laar [10] who studied reproductive rights and options available to women infected with HIV in Ghana: perspectives of service providers from three Ghanaian health facilities, who found in her study majority of studied sample were married and Most of the students' had incorrect knowledge about services of reproductive health relation to component of reproductive health services of childhood, reproductive health services of teenage, and reproductive health services of menopause in pre intervention. After Instruction booklet implementation there were improved knowledge and highly statistical difference in both immediate post intervention and eight weeks post intervention than pre intervention. This mean be due to inadequate educative program about reproductive health services. These findings were agreement with Ibrahim [11], who found that most of the adolescents' girl know the major service of reproductive health and have improve in knowledge after application of guideline. Similarly, this finding was agreement with Hlatshwayo [12], who studied Exploring adolescents' perceptions regarding youth-friendly sexual and reproductive health services in a selected Community Health Centre (CHC) in uMgungundlovu District. He reported that the majority of adolescents still lack awareness and autonomy to access sexual reproductive health related information and services.

As regards to nursing students' knowledge about objectives of premarital counseling. The present study reveals that all of studied sample had correct knowledge and highly statistically significant improvement in knowledge regarding premarital counseling at immediate post intervention and eight weeks post intervention. This may be due to minor role of peer in giving knowledge about health services that provide premarital counseling. These finding were agreeing with Ibrahim, et al. [13] who found in their study about Premarital Counseling program: knowledge, attitude, and satisfaction of attendees of governmental outpatient clinics in Jeddah that there was highly statistically significant improvement in knowledge regarding premarital counseling at different two phases of assessment after program implementation rather than students' knowledge were poor during pretest.

Regarding nursing students' knowledge about of female genital mutilation the finding of the present study illustrates that the majority of nursing students' had incorrect knowledge pre intervention and had improvement immediate post intervention and 8 weeks post intervention. These findings supported by
Kroon and Binsalamah [14], They revealed that most of studied sample were had inadequate knowledge regarding female genital mutilation and its complication during pretest while there were highly statistical significant differences between knowledge mean scores pre intervention, immediately post intervention and three weeks post intervention. While finding disagrees with Lee \& Strong [15], they reported that women with FGM during labor were at higher risk of obstetrical complications than women without FGM. This may be due to inadequate health education regarding FGM to students' and minor role of university staff toward students' heath education.

According to the nursing students' knowledge regarding age at marriage the present study pointed out that only about half of the students had answer that 18 years old was the appropriate age of marriage. This finding was supported by Ahmed, [16], who found that the majority of the commercial and industrial school's students' gave wrong answer regarding appropriate age of marriage. Also disagree with Kaphle [17], who found that most of studied sample were aware about the right marriage age of $\geq 20$ years.

Regarding to nursing student's knowledge related to sexually transmitted diseases, the present study reveals that the majority of studied sample had incorrect knowledge during pre-intervention and were had improvement of nursing students' knowledge immediately post intervention and 8 weeks post intervention rather than pre intervention.

These findings come in agreement with Fuadi [18], study aimed to examine and link learning culture-based human reproductive system in order to improve understanding of reproduction and prevent reproductive disease. This finding disagree with Abajobir and Seme [1] they studied reproductive health knowledge and services utilization among rural adolescents in east Gojjam zone, Ethiopia: a community-based cross-sectional study, Ethiopia, they revealed that two-third of the adolescents had ever heard about sexually transmitted diseases and mode of transmitions.

In relation to nursing female's students' knowledge about concept of reproductive rights the present study finding were reveals that the majority of studied sample had incorrect knowledge pre-intervention and had improvement of knowledge immediate post intervention and after 8 weeks post intervention. Also agree with Panday, et al. [19], who studied the contribution of female community health volunteers FCHVs) to maternity care in Nepal and Laar [10] who studied reproductive rights and options available to women infected with HIV in Ghana who reported that least of them know about women's right. On the other hand, finding were disagree with Dida, et al. [20], they studied Reproductive health services utilization and its associated factors among Madawalabu University Students. They mentioned that majority of the respondents were had correct meaning of reproductive rights.

Regarding to nursing student's knowledge about woman right to life, freedom, security and privacy the present study revealed 
that most of studied sample were had incorrect knowledge pre-intervention and had improvement of knowledge during immediate post intervention and eight weeks post intervention. This finding was disagree with Shailaja, [21], who studied the importance of patient privacy during a clinical examination of individuals regarding their personal health information being used or disclosed without their consent. Also finding disagree with Center for Disease Control and Prevention [22], they reported that the rights to life and health are central to the enjoyment of all other human rights, and fulfillment of this right requires governments to take steps to reduce maternal mortality and increase life expectancy.

Regarding to students' nurse knowledge about woman right to avoid exposure to false believes as female genital mutation the present study reveals that majority of studied sample had incorrect knowledge during pre-intervention intervention and had improvement of knowledge during immediate post intervention and 8 weeks post intervention. This finding in similarly with Dixon et al. [23], they studied Tackling female genital mutilation in the UK, they found that here are growing numbers of women living in the UK who have experienced female genital mutilation/ cutting. Evidence is needed to understand how best to meet their health-care needs and to shape culturally appropriate. Also agree with Gebre et al. [24] who found that female genital mutilation is a traditional harmful practice that violates the rights and dignity of women and girls. Regarding to nursing student's knowledge about woman right to health the present study shows that majority of studied sample had incorrect knowledge during preintervention and had improvement of knowledge in immediate post intervention and 8 weeks post intervention. This finding was agreed with Hallila, \& Al-Halabi [25], they studied Saudi female university employee self-determination in their own healthrelated issues and revealed that most of the participants did not fully understand the health rights of women in Saudi Arabia.

Regarding to nursing student's knowledge about woman right to marriage and found a family the present study revealed that more than two thirds of studied sample had incorrect knowledge during pre-intervention and had improvement of knowledge during immediate post intervention and three weeks post intervention. This finding disagree with Rashad, et al. [26] \& Khosla [27], they reported that the majority of the study sample agrees that girls have the right to choose their prospective husband and marry. Similarly, this finding disagree with Germain, et al. [28], who found that most of the total respondents were aware about the right marriage age of $\geq 20$ years.

Regarding to nursing student's knowledge about woman right to healthy and secure pregnancy the finding of the present study reveals that most of studied sample had incorrect knowledge during pre-intervention and had improvement of knowledge during immediate post intervention and 8 weeks post intervention.
This finding disagree with Kismödi, et al. [2], they mentioned that respect, protect and fulfill human rights in relation to maternal health, pregnancy and childbirth.

Regarding to nursing student's knowledge about woman right to family planning the present study revealed that most of studied sample had incorrect knowledge during pre-intervention and had improvement of knowledge during immediate post intervention and eight weeks post intervention. This may be due to students not aware about woman right to decide the number of children and spacing between pregnancies. This finding is in-accordance with Rashad, et al. [23], they reported that the majority of respondents were aware about their right to decide the number and spacing of their children. In relation to nursing student's knowledge about woman rights to non-discrimination the finding of the present study reveals that majority of studied sample had incorrect knowledge during pre-intervention and had improvement of knowledge during immediate post intervention and eight weeks post intervention. This change in knowledge may be due to application of guide line.

This finding consistent with Norton [29], they found that women should be equal to men and that both sexes should have equal rights to decide over their own sexuality. Finding disagree with Coker, et al. [30], has found that female-identified youth and sexual minorities are less. Regarding relation between studied nurses' general characteristics and mean score of studied nurses' knowledge related to women reproductive rights pre immediately post 8 weeks' post. No statistically significant difference was found. This finding disagrees with Cameron [31], who pointed out that there was a positive relation between knowledge of the studied students and their age.

In relation between nursing student's level of knowledge and mother education. The finding of the present reveals that there was highly statistically significant difference $(P \leq 0.001)$ between nursing student's level of knowledge and mother education. This finding agrees with Ibrahim, [11] who found positive relation between students' mother educational level and total knowledge. This finding agrees with DeBeaudrap [32], who found positive relation in their study about Disability and Access to Sexual and Reproductive Health Services in Cameroon: A Mediation Analysis of the Role of Socioeconomic Factors also, in the same line with Ajara \& Shuaib [33], who study Knowledge and sociodemographic data among reproductive-age women (RAW) in Malete, Kwara State [34].

\section{Conclusion}

In the light of study findings, it was concluded that there was a highly statistically significant improvement in nursing students' knowledge and concept after application of Instruction booklet about woman rights in reproductive health. This supported the main hypothesis of the study. 
In the light of study findings, the following recommendations can be suggested:

a) Periodic awareness program for nursing students about reproductive health and right.

b) Mass media sector is required to broad cast well designed messages related to reproductive rights to all community.

c) Nusing education institutions should strengthen and upgrade the reproductive health rights and counseling components into their curricula.

\section{d) Further studies}

The study should be applied on parents to evaluate their knowledge regarding reproductive health and rights.

\section{References}

1. Abajobir A, Seme A (2014) Reproductive health knowledge and services utilization among rural adolescents: community-based crosssectional study, Ethiopia. BMC Health Serv Res 28(14): 139.

2. Kismödi E, Cottingham J, Gruskin S, Miller AM (2015) Advancing sexual health through human rights: The role of the law. Global public health 10(2): 252-267?.

3. World Health Organization (2014) Health for the world's adolescents A second chance in the second decade Department of Maternal N (Ed.), Child and adolescent health. World Health Organization, Geneva.

4. Herbst N (2017) Sexual and Reproductive Health and Rights Education Sex Education in The Digital Era, p. 43.

5. Santa Maria D, Guilamo-Ramos V, Jemmott LS, Derouin A, Villarruel A, et al. (2017) Nurses on the Front Lines: Improving Adolescent Sexual and Reproductive Health Across Health Care Settings: An evidencebased guide to delivering counseling and services to adolescents and parents. Am J Nurs 117(1): 42-51.

6. Black RE, Laxminarayan R, Temmerman M, Walker N (2016) Reproductive maternal newborn and child health. Disease control priorities, 2.]

7. Marchant T, Bhutta ZA, Black R, Grove J, Kyobutungi C, et al. (2019) Advancing measurement and monitoring of reproductive, maternal, newborn and child health and nutrition: global and country perspectives. BMMJ Global Health 4(suppl 4).

8. Kaur G, Sharma DK, Joshi U (2017) An experimental study to assess the effectiveness of mint leaves paste on dysmenorrhea among nursing students at sgrd college of nursing, vallah, Amritsar, Punjab. International Journal of Development Research 7(07): 14208-14212.]

9. Govender D, Naidoo S, Taylor M (2019) Knowledge, attitudes and peer influences related to pregnancy, sexual and reproductive health among adolescents using maternal health services in Ugu, KwaZulu-Natal, South Africa. BMC public health 19(1): 928.]

10. Laar AK (2013) Reproductive rights and options available to women infected with HIV in Ghana: perspectives of service providers from three Ghanaian health facilities. BMC women's health 13(1): 13.'

11. Ibrahim A (2010) Effect of Educational Program on Adolescent Girls Regarding Reproductive Health. Benha University, Egypt.

12. Hlatshwayo KL (2017) Exploring adolescents' perceptions regarding youth-friendly sexual and reproductive health services in a selected Community Health Centre (CHC) in uMgungundlovu District (Doctoral dissertation)?
13. Ibrahim NK, Bashawri J, Al Bar H, Al Ahmadi J, Al Bar A, et al. (2013) Premarital screening and genetic counseling program: knowledge, attitude, and satisfaction of attendees of governmental outpatient clinics in Jeddah. J Infect Public Health 6(1): 41-54.'

14. Kroon S, Binsalamah S (2015) Nursing students' views on female genital mutilation in Tanzania. Journal of Midwifery \& Women's Health 58(4): 451-456.

15. Lee M, Strong N (2015) Female genital mutilation: What ob/gyns need to know. Contemporary OB/GYN 60(11): 20-24.

16. Ahmed M (2010) Premarital Counseling for Late Adolescent Females. Benha University, Egypt.

17. Kaphle M (2013) Awareness and utilization of reproductive rights among the women of reproductive age in kapan VDC, Nepal. HAS 3(1): 5-9.

18. Fuadi $\mathrm{T}$ (2019) Reproduction based on islamic culture: effort to increase understanding of reproduction system and prevention of infectious diseases. Journal Ilmiah Peuradeun 7(2): 269-284.]

19. Panday S, Bissell P, Van Teijlingen E, Simkhada P (2017) The contribution of female community health volunteers (FCHVs) to maternity care in Nepal: a qualitative study. BMC health services research 17(1): 623.]

20. Dida N, Darega B, Takele A (2015) Reproductive health services utilization and its associated factors among Madawalabu University students, Southeast Ethiopia: cross-sectional study. BMC research notes 8(1): 8]

21. Shailaja T (2007) The importance of patient privacy during a clinical examination, Indian J Med Ethics 4(2): 65.

22. Center for Disease Control and Prevention (2014) ICPD and human rights.

23. Dixon S, Shacklock J, Leach J (2019) Tackling female genital mutilation in the UK: Female genital mutilation: barriers to accessing care. The BMJ 364: 1921.'

24. Gebre T, Hagos F, Teklu G, Fisseha M, Abera M, et al. (2019) The prevalence of gender-based violence and harmful traditional practices against women in the tigray region, Ethiopia. Journal of Asian and African Studies 55(1): 58-75.]

25. Hallila LE, Al-Halabi JO (2018) Saudi female university employee selfdetermination in their own health-related issues. Journal of Nursing Education and Practice 8(8).

26. Rashad W, Shamekh A, Ragheb S (2013) Assessment of women's awareness about reproductive rights. American Journal of Research Communication 1(9): 65-83.

27. Khosla R, Zampas C, Vogel JP, Bohren MA, Roseman M, et al. (2016) International human rights and the mistreatment of women during childbirth. Health hum rights 18(2): 131-143.

28. Germain A, Sen G, Garcia-Moreno C, Shankar M (2015) Advancing sexual and reproductive health and rights in low-and middle-income countries: Implications for the post-2015 global development agenda. Global Public Health 10(2): 137-148.

29. Norton A (2020) On the Muslim question (Vol. 19). Princeton University Press, USA.

30. Coker AL, Bush HM, Clear ER, Brancato CJ, McCauley HL, et al. (2020) Bystander program effectiveness to reduce violence and violence acceptance within sexual minority male and female high school students using a cluster RCT. Prevention Science 21: 434-444.

31. Cameron A, Smith E, Mercer N, Sundstrom B (2020) 'It is our duty: 'understanding parents' perspectives on reproductive and sexual health education. Sex Education 20: 1-17. 
32. DeBeaudrap P, Mouté C, Pasquier E, Mac-Seing M, Mukangwije PU, et al. (2019) Disability and access to sexual and reproductive health services in cameroon: a mediation analysis of the role of socioeconomic factors. Int J Environ Res Public Health 16(3): 417.

33. Ajara TA, Shuaib QA (2019) Knowledge and Attitude to Sexual and Reproductive Health Rights among Reproductive-Age Women (RAW) in Malete, Kwara State. Global Journal of Management and Business Research. On mental research and public health 16(3): 417.

34. Miller A, Gruskin S, Cottingham J, Kismödi E (2015) "Sound and Fury engaging with the politics and the law of sexual rights." Reproductive Health Matters 23(46): 7-9.

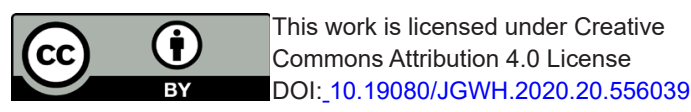

\section{Your next submission with Juniper Publishers will reach you the below assets}

- Quality Editorial service

- Swift Peer Review

- Reprints availability

- E-prints Service

- Manuscript Podcast for convenient understanding

- Global attainment for your research

- Manuscript accessibility in different formats

( Pdf, E-pub, Full Tsext, Audio)

- Unceasing customer service

Track the below URL for one-step submission https://juniperpublishers.com/online-submission.php 